\title{
Photoinduced birefringence and diffraction efficiency in azo dye doped or grafted polymers: theory versus experiment of the temperature influence
}

\author{
Blanche P-A ${ }^{(1)}$, Lemaire Ph. $\mathrm{C}^{(1)}$ Maertens $\mathrm{C}^{(2)}$, Dubois $\mathrm{P}^{(2)}$, Jérôme $\mathrm{R}^{(2)}$ \\ ${ }^{(1)}$ Centre Spatial de Liège, Université de Liège, Parc Scientifique du Sart Tilman, Avenue du Préaily, \\ B-4031 Angleur-Liège, Belgium \\ ${ }^{(2)}$ Centre for Education and Research on Macromolecules, Universite de Liège, Sart Tilman B6a, \\ B-4000 Liège, Belgium
}

\begin{abstract}
We have investigated the influence of the temperature on the photoinduced birefringence as well as on the diffraction efficiency of azo dye doped or grafted polymers. The samples are composed of three polymer matrices containing 2, 5-dimethyl-4-( $p$-nitrophenylazo anisole). We propose two theoretical models to explain the experimental increase of both phenomena when the temperature is decreased. Models are based on the statistical angular distribution of the chromophores that depends on the intensity of the polarized laser beam in the media, counteracted by the thermal agitation. Parameters introduced in both models can be used to characterize the polymeric system properties.
\end{abstract}

\section{INTRODUCTION}

Interest in polymeric compounds that reversibly change their refractive index with illumination has increased in the past few years. The stimulus for these investigations is the potential application of these kinds of materials in photonic devices, whose importance is growing in manyfields. ${ }^{[1-7]}$ Azo dye molecules can be redirected by the electric field of polarized light. This arises by multiple trans-cis pho-toisomerizations of the nitrogen-nitrogen double bound, followed by cis-trans backthermal relaxation. The isomerizations allow the molecules to turn until their transition axis is placed perpendicularly to the light polarization axis ${ }^{\left[{ }^{8-10]}\right.}$ This phenomenon leads to macroscopic effects such as photodichroism and photoinduced birefringence, since the complex refractive index is different along or perpendicular to the transition axis. ${ }^{[10-14]}$ With the use of polarized laser beams, both effects can be used to write absorption or phase holograms in these kinds of media. ${ }^{[7,8,13,15]}$

The temperature dependence of the mechanical characteristics of plastics has already been studied for a long time. On the other hand, there is only limited information regarding the influence of the temperature on the optical properties in these materials. ${ }^{[10,16-18]}$ However, it is important to understand how parameters modify the sensitivity as well as the amplitude of the photoinduced birefringence and the diffraction efficiency. Indeed, they are figures of merit that must be optimized for further applications. In this paper we provide experimental measurements as well as theoretical treatments of the temperature influence on the amplitude of photoinduced birefringence and on the diffraction efficiency of phase holograms written in azo dye doped polymers. The fit of the experimental data by theoretical models has allowed the determination of parameters that characterize the polymer behavior for objective comparison between compounds. In addition, as the models use the same parameters, we can obtain validation of the whole development. Experiments have been carried out on films containing 15 weight percent (wt.\%) of the 2, 5-dimethyl-4-( $p$-nitrophenylazoanisole) (DMNPAA) azo dye. Indeed, we have shown, in a previous paper, that photoinduced orientation of this chromophore can occur. The first compound is a mix of the birefringent molecules with poly ( $N$-vinyl carbazole) (PVK). This compound has a particular importance, since it is well-known to be a good photorefractive polymer if $1 \mathrm{wt} . \%$ of (trinitrofluor-enone) is added. ${ }^{[19,20]}$ The two other matrices are copolymers of [ $\omega$ ( $N$-carbazolyl)alkylmethacrylate] and [4-(11-methacryloylalkyloxy)-2, 5-dimethylphenyl](4nitrophenyl) diazene. Both alkyl spacer lengths have been set to be hexyl and undecyl for C6-C11DMNPAA and undecyl and hexyl for C11-C6-DMNPAA. The chemical structure of these compounds is presented in Fig. 1. Glass transition temperatures $\left(T_{g}\right)$ have been measured by differential scanning calorimetry to be equal to $288 \mathrm{~K}$ for PVK:DMNPAA, $329 \mathrm{~K}$ for C6-C11-DMNPAA, and $253 \mathrm{~K}$ for C11-C6-DMNPAA. Polymer and chro-mophore synthesis can be found elsewhere. 


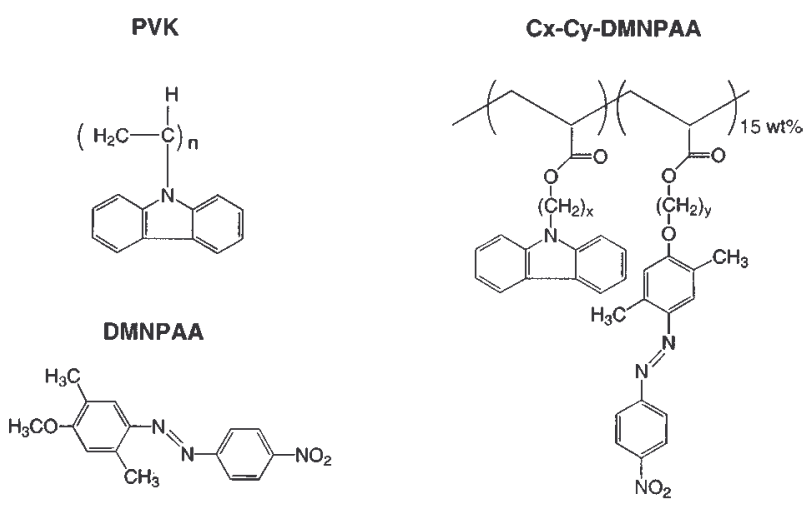

Fig. 1. Chemical structures of the different compounds used, along with their acronyms. The chromophore DMNPAA is always add in $15 \mathrm{wt} \%$ to the polymer matrix.

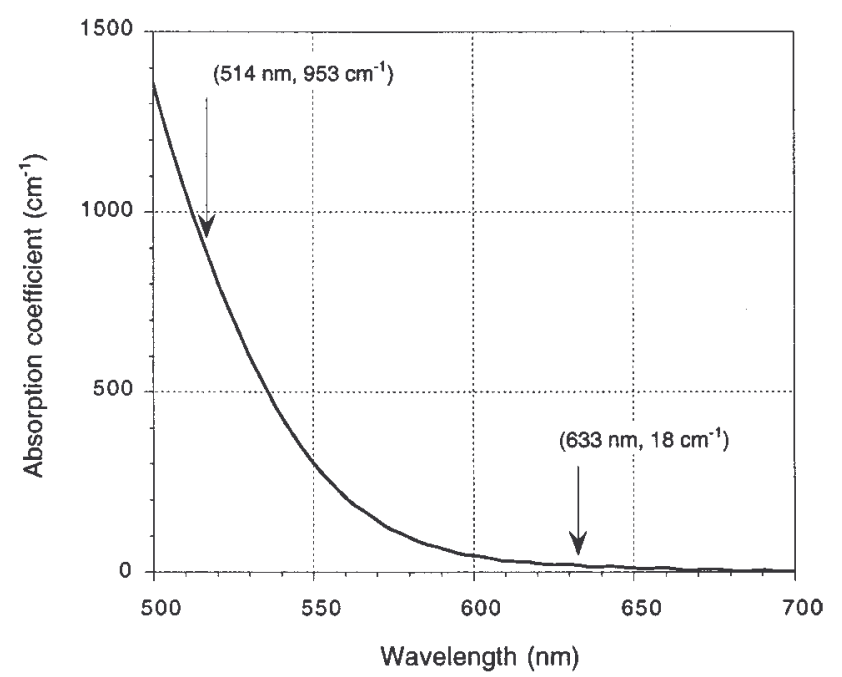

Fig. 2. Absorption coefficient spectrum of a 15-wt.\% DMNPAA doped PVK polymer film. The absorption coefficients of both working wavelengths are indicated by arrows.

Samples have been made by pressing down polymer powder on two glass plates and by heating these plates $20^{\circ} \mathrm{C}$ above the polymer $T_{g}$. The sample thickness is determined by $70-\mu \mathrm{m}$ spacers. Cooling happens at open air and ambient temperature. Since crystallization has already been observed with high DMNPAA concentration (50 wt.\%), no film degradation has been noted during three years with our 15wt.\% compounds. The thermal cyclings done for this work have not altered properties of the samples. Theoretical models proposed in this paper are based on the statistical angular distribution of the birefringent molecules. Two processes are counteracting to form this distribution: The polarized laser light attempts to align the molecules, whereas the thermal agitation randomizes their orientation. Since the sample has important absorption coefficients at the working wavelengths (see Fig. 2), we have introduced into our models the effects of the decreasing intensity of the laser light through the polymer film. In this way we attempt to approach realistic conditions of illumination in the media.

\section{PHOTOINDUCED BIREFRINGENCE}

\section{A. Experiments}

The experimental setup used to study the influence of the temperature on the photoinduced birefringence is shown in Fig. 3. A more detailed graphic has already been presented in Ref. [22]. To align the dye molecules, an argon laser beam $(514 \mathrm{~nm})$ is filtered, expanded, and linearly polarized before going through the sample. Its intensity has been set to $2.1 \mathrm{~mW} / \mathrm{cm}^{2}$. A He-Ne laser beam (633 $\mathrm{nm}$ ) is used as a probe to analyze the chromophore alignment. Its polarization is rotated $45^{\circ}$ with respect to the argon beam. After having crossed the sample, the He-Ne beam goes through an analyzer turned $90^{\circ}$ with respect to its initial polarization. The intensity of the He-Ne beam has been selected 
low enough not to influence the orientation of the DMNPAA molecules $\left(0.1 \mathrm{~mW} / \mathrm{cm}^{2}\right)$. The sample is placed on a thermal regulation device that allows its temperature to be set between the ambient temperature $(300 \mathrm{~K})$ and $250 \mathrm{~K}$. To reach this temperature without condensation problems, the sample is put inside a vacuum chamber.

Without a pumping beam (argon), chromophore molecules are in principle randomly oriented. The sample is centrosymmetric and cannot exhibit any birefringence. In these conditions the He-Ne laser does not undergo any polarization modification when crossing the sample and so is completely stopped by the analyzer. When the ar-gon beam is switched on, the azo molecules begin to rotate by multiple trans-to-cis photoisomerizations and cis-trans backthermal relaxations. Chromophores rotate until they are placed perpendicularly to the argon polar-ization. Indeed, photoisomerizations take place until the transition axes of the molecules are placed perpendicu-larly to the argon polarization: In such a position, the la-ser light cannot stimulate the electronic transition any longer. Since the dielectric constant of the azo molecules is different according to whether they are along the tran-sition axis or in the perpendicular plane, the sample ex-hibits birefringence when chromophores are aligned. The HeNe polarization is modified when crossing the polymer film, and its component perpendicular to the analyzer axis can reach the detector. The transmission efficiency is calculated as the ratio of the initial $\mathrm{He}-\mathrm{Ne}$ power to the power detected after the analyzer.

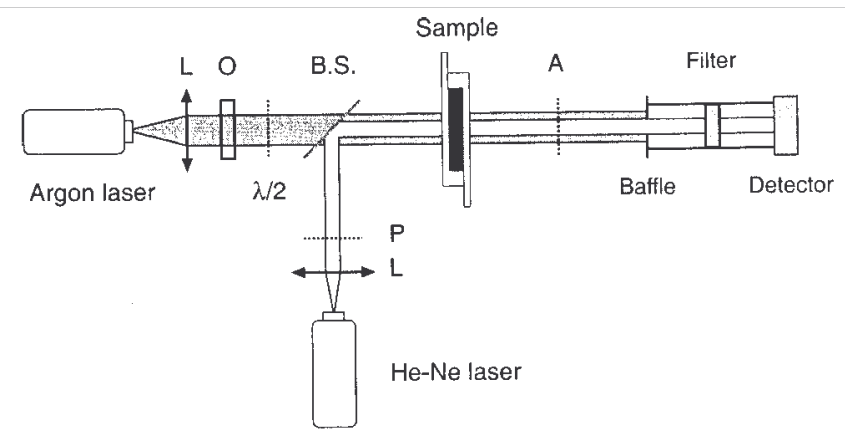

Fig. 3. Photoinduced birefringence setup. L, lens; O, shutter; $\lambda / 2$, half-wave plate; B.S., beam splitter; $P$, polarizer; A, analyzer.

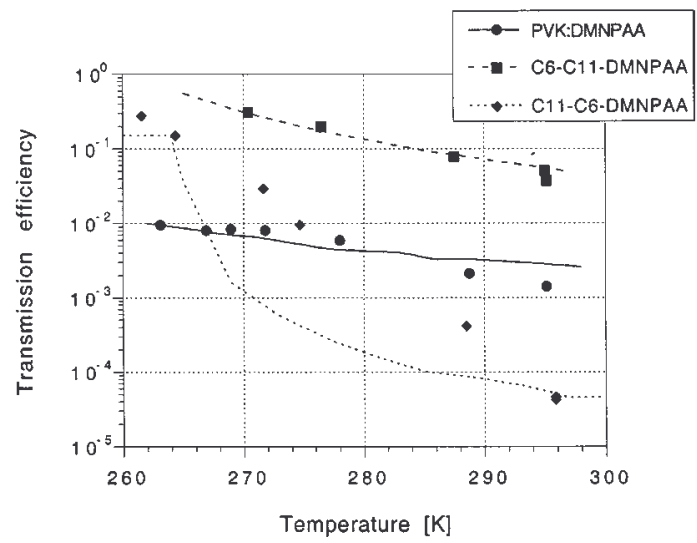

Fig. 4. Saturation amplitude of the transmission efficiency versus temperature of the sample for the three polymers studied. Curves are interpolations by the theoretical model. 


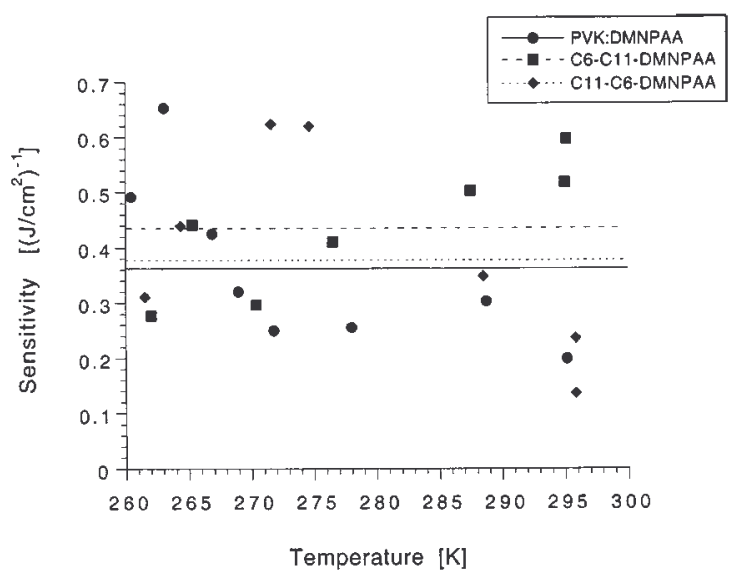

Fig. 5. Sensitivity of the photoinduced birefringence versus sample temperature for the three polymers studied.

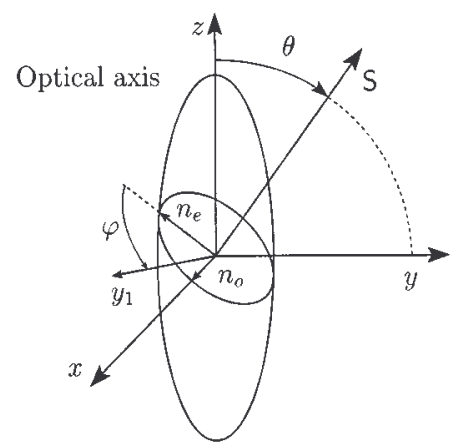

(a)

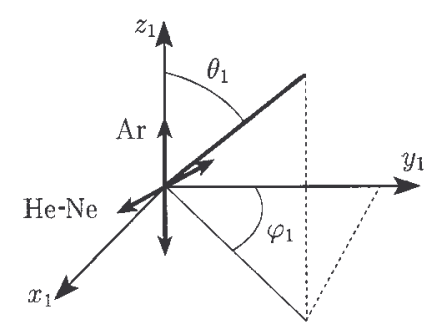

(b)

Fig. 6. Geometry of the coordinate system axes used in the theoretical models: (a) coordinates associated with a birefringent element and (b) coordinates associated with the laboratory. See the text for the definitions of axes and vectors.

The experimental curves of transmission efficiency as a function of time have been fitted by the biexponential function included in a square sine:

$$
\begin{aligned}
\eta(t)= & \sin ^{2}\left(A\left[1-\exp \left(-t / \tau_{A}\right)\right]\right. \\
& \left.+B\left[1-\exp \left(-t / \tau_{B}\right)\right]+\phi\right) .
\end{aligned}
$$

The phase parameter $\varphi$ is defined by the transmitted intensity before the writing beam is switched on. This comes from the sample-making process, which aligns some chromophores and so induces some " "natural"' birefringence. That function, without the square sine, has already been used by many other authors ${ }^{[7,16,18,23,24]}$ and found to be the simplest curve that fits this data type. However, it must be noted that there exists a complete theory describing the kinetics of the molecular reorientation that has proved to be able to fit that measurement ${ }^{[14,25]}$. Since our goal was not to describe the dynamics of the signal, we have preferred to use the biexponential function for the sake of simplicity.

According to Eq. (1), the maximum photoinduced bire-fringence is given by the efficiency at saturation minus the efficiency at $t=0$ : 


$$
\eta_{\max }=\sin ^{2}(A+B) .
$$

In Fig. 4 we have plotted the maximum photoinduced birefringence as a function of the temperature. One can see that, for the three compounds, the transmission efficiency increases considerably as the temperature de-creases. We ascribe this behavior to the thermal agitation, which prevents the molecules from being perfectly aligned by the electric field of the argon laser beam.

It is also interesting to analyze the behavior of the sensitivity versus the sample temperature (Fig. 5). Sensitivity is commonly related to the slope of the refractive-index variation [argument of the square sine function (1), ${ }^{[2]}$ it-self proportional to the chromophore photo-orientation] at the origin time divided by the writing intensity. Sensitivity is thus given by

$$
S=\frac{\frac{A}{\tau_{A}}+\frac{B}{\tau_{B}}}{E_{\mathrm{Ar}}\left[1-\exp \left(-\alpha_{\mathrm{Ar}} d\right)\right]} .
$$

\section{B. Mathematical Model}

To describe the behavior of transmission efficiency versus temperature, we will consider the sample to be composed of an important number of birefringent elements. Each possesses the same index ellipsoid but has different orientations. These elements are abstract representations but can be assimilated to a small group of azo molecules, which are all oriented in the same direction. The index ellipsoid of such an element can be assimilated as a revolution one, since the DMNPAA dielectric coefficient along the transition axis is very different from those along other axes.

Both coordinate systems used in the mathematical model are presented in Fig. 6. The first [Fig. 6(a)] is as-sociated with a birefringent element. The axis $z$ is along the optical axis of the birefringent element. The vector $\mathbf{S}$ is along the direction of propagation of the reading He-Ne light, and it is included in the $y-z$ plane (this defines the $y$ axis). $n_{e}$ is perpendicular to $n_{o}$, and both are included in a plane orthogonal to the $\mathbf{S}$ vector. The second system [Fig. 6(b)] is associated with the laboratory: $x_{1}$ is along the laser beam's direction of propagation, and $z_{l}$ is along the argon polarization. $\theta_{1}$ and $\boldsymbol{\varphi}_{l}$ are the Euler angles defining the orientation of the birefringent element opti-cal axis.

When the He-Ne beam goes through one of these bire-fringent elements, its initial polarization is modified and can be described, in the Jones formalism, by

$$
\left(\begin{array}{l}
E_{x}^{\prime} \\
E_{y}^{\prime}
\end{array}\right)=\exp \left(-\frac{\alpha_{\mathrm{He}-\mathrm{Ne}} d}{2}\right)\left[R_{(-\varphi)} \times W_{0}+R_{(\varphi)}\right] \times\left(\begin{array}{l}
E_{x} \\
E_{y}
\end{array}\right)
$$

where $a$ is the coefficient of absorption at the He-Ne wavelength $(633 \mathrm{~nm})$ and $d$ is the thickness of a birefrin-gent element. $E_{i}$ is the incident light electric field with respect to the $i$ direction, $R\left(_{\varphi}\right)$ is the rotational matrix, and $W_{0}$ is the Jones matrix describing the phase delay. The definitions of $R(\varphi)$ and $W_{0}$ are given by

$$
\begin{aligned}
R_{(\varphi)} & =\left[\begin{array}{cc}
\cos \varphi & \sin \varphi \\
-\sin \varphi & \cos \varphi
\end{array}\right], \\
W_{0} & =\left[\begin{array}{cc}
\exp \left(-i \frac{\Gamma}{2}\right) & 0 \\
0 & \exp \left(i \frac{\Gamma}{2}\right)
\end{array}\right] .
\end{aligned}
$$

$\boldsymbol{\varphi}$ is the angle between the He-Ne polarization axis and the vector $n_{e}$ defined in Fig. 6(a). $\boldsymbol{T}$ is the phase delay of a birefringent element and is expressed as follows:

$$
\Gamma=\frac{2 \pi}{\lambda} d\left[n_{o}-n_{e}(\theta)\right]
$$

where $\lambda$ is the wavelength of the He-Ne beam $(633 \mathrm{~nm})$ and $n_{o}$ and $n_{e}(\theta)$ are the ordinary and the extraordinary index of refraction in the plane perpendicular to the He-Ne propagation axis. $\boldsymbol{\theta}$ is defined in Fig. 6(a) as the angle formed by the optical axis of the birefringent element and the direction of propagation of the He-Ne beam.

The extraordinary index used in Eq. (6) depends on the orientation of the birefringent element. Thus, for the geometry presented in Fig. 6(a), this index is given by 


$$
\frac{1}{n_{e}^{2}(\theta)}=\frac{\cos ^{2} \theta}{n_{o}^{2}}+\frac{\sin ^{2} \theta}{n_{e}^{2}},
$$

where $n_{e}$ is the extraordinary index of refraction of the bi-refringent elements.

When the He-Ne beam goes through the sample, it crosses a great number $(N)$ of birefringent elements. The longitudinal and transversal electric fields at the output of the sample are given by the following matrix equation, where each square bracket stands for one element:

$$
\begin{aligned}
\left(\begin{array}{l}
E_{x}^{\prime} \\
E_{y}^{\prime}
\end{array}\right)= & \exp \left(-\frac{N \alpha_{\mathrm{He}-\mathrm{Ne}} d}{2}\right)\left[R_{\left(-\varphi^{N}\right)} \times W_{0}^{N} \times R_{\left(\varphi^{N}\right)}\right] \\
& \times\left[R_{\left(-\varphi^{N-1}\right)} \times W_{0}^{N-1} \times R_{\left(\varphi^{N-1}\right)}\right] \\
& \times \cdots \times\left[R_{\left(-\varphi^{1}\right)} \times W_{0}^{1} \times R_{\left(\varphi^{1}\right)}\right] \times\left(\begin{array}{l}
E_{x} \\
E_{y}
\end{array}\right) .
\end{aligned}
$$

Not all the birefringent elements are oriented according to the same Euler angles defined in Fig. 6(b). Indeed, the thermal agitation randomizes the molecular alignment that the argon beam tries to impose. Therefore there is no relationship between successive angles, and it is not possible to reduce the number of matrices present in Eq. (8), as could be done for a Solc filter or for a chiral nematic liquid crystal. To determine the angles $\theta^{i}$ and $\varphi^{i}$ to insert into Eq. (8), we are going to use the angular distribution of the birefringent elements as a function of macroscopic parameters (argon beam intensity, sample temperature). In this way it will be possible to carry out a Monte Carlo simulation of the light electric field propagation inside the sample.

The system is composed of $N$ classical bodies. Thus the Maxwell-Boltzmann statistic can be applied to describe the angular distribution. The following equation gives the number of elements with their optical axis orientated between $\theta_{1}$ and $\theta_{1}+\mathrm{d} \theta_{1}$ and between $\varphi_{1}$ and $\varphi_{1}+d \varphi_{1}$ as a function of the temperature $T$ :

$$
\begin{aligned}
n b\left(\theta_{1}, \varphi_{1}\right) \mathrm{d} \theta_{1} \mathrm{~d} \varphi_{1} \\
=\frac{N \exp \left[\frac{-E\left(\theta_{1}, \varphi_{1}, T\right)}{k T}\right]}{z\left(\theta_{1}, \varphi_{1}\right)} \omega\left(\theta_{1}, \varphi_{1}\right) \mathrm{d} \theta_{1} \mathrm{~d} \varphi_{1},
\end{aligned}
$$

where $z\left(\theta_{1}, \varphi_{1}\right)$ is the partition function $\operatorname{and} E\left(\theta_{1}, \varphi_{1}, T\right)$ is the energy of the molecule in the state $\left(\theta_{1}, \varphi_{1}\right)$ at temperature T. $\omega\left(\theta_{1}, \varphi_{1}\right)$ is the statistical weight associated with the space direction $\left(\theta_{1}, \varphi_{1}\right)$; in spherical coordinates it is defined as $\omega\left(\theta_{1}, \varphi_{1}\right)=\left(\sin \theta_{1}\right) / 2$.

As a statistical distribution, Eq. (9) is subject to the general conditions of normalization, namely,

$$
\begin{array}{r}
\iint_{0}^{2 \pi} n b\left(\theta_{1}, \varphi_{1}\right) \mathrm{d} \theta_{1} \mathrm{~d} \varphi_{1}=N, \\
\iint_{0}^{2 \pi} E\left(\theta_{1}, \varphi_{1}\right) n b\left(\theta_{1}, \varphi_{1}\right) \mathrm{d} \theta_{1} \mathrm{~d} \varphi_{1}=E,
\end{array}
$$

which allow one, by inserting Eq. (9) into Eq. (10), to calculate the partition function to be

$$
z\left(\theta_{1}\right)=\int_{0}^{\pi} \exp \left[\frac{-E\left(\theta_{1}, \varphi_{1}, T\right)}{k T}\right] \frac{\sin \theta_{1}}{2} \mathrm{~d} \theta_{1} .
$$

In Subsection 2.A we have discussed the fact that chro-mophores tend to redirect themselves perpendicularly to the polarization axis of the argon beam. The trans-cis photoisomerization can occur only if the molecule has a component of its dipolar axis along the electric field of the argon beam. The energy of the molecules and thus one of the birefringent elements can be described by a squared cosine of the angle between the optical axis and the argon polarization ${ }^{[14,27,28]}$ :

$$
E\left(\theta_{1}, T\right)=A(T) \epsilon \cos ^{2} \theta_{1},
$$

where $\varepsilon$ is the amplitude of the argon beam electric field at the element place and $A(T)$ is a function depending on various molecular constants as well as on the sample temperature. We will now discuss this function in detail.

The Maxwell-Boltzmann distribution describes the angular orientation as a function of the temperature given that there is no transformation in the material. This is not exact in the polymers, since phase transitions can happen or a continuous variation of the constraints can be applied to the chromophores. To take the latter into account, we have modified the Maxwell-Boltzmann distribution by introducing the $A(T)$ function into the potential energy equation. $A(T)$ has the following form: 


$$
A(T)=A \frac{T}{T-T_{0}},
$$

where $A$ is a parameter depending on the number of molecules in a birefringent element as various molecular constants (cross section, dipolar momentum, quantum efficiency). This parameter reflects the ability of the molecules to reorient under the illumination effect; thus it also depends on the interactions between the polymer matrix and the chromophores. This could not be constant with the temperature, but since we will not introduce the molecular parameters, the thermal dependence will be included only in the $A(T)$ function and will not be split into its various components (also for the sake of simplicity). The $A$ dimension is energy divided by an electric field: $\mathrm{J}(\mathrm{V} / \mathrm{cm})^{-1}$.

$T_{0}$ is a temperature threshold; at this temperature value, all the molecules are perfectly oriented, and, furthermore, decreasing the temperature does not influence the distribution any longer. So Eq. (14) is valid only for $T>T_{0}$. To our knowledge, we think that this parameter is related to the free volume of the polymer. Indeed, the latter varies with the temperature. $T_{0}$ stands for the temperature at which the free volume of the polymer is small enough to avoid thermal disorientation of the chromophores. Equation (14) can also be understood as the introduction of the Arrhenius theory as well as the William-Landel-Ferry theory ${ }^{[33,34]}$ in our model.

Crossing the sample, the argon beam is strongly absorbed. The amplitude of the electric field used in Eq. (13) is thus not constant over the thickness of the polymer film. Let $\alpha$ be the absorption coefficient of the film at $514 \mathrm{~nm}$; thus $e$ is varying with the sample depth as

$$
\epsilon=\epsilon_{0} \exp \left(-\frac{\alpha_{\mathrm{Ar}} L}{2}\right) .
$$

By inserting Eqs. (12)-(15) into the definition of the angular distribution, we obtain, in laboratory coordinates,

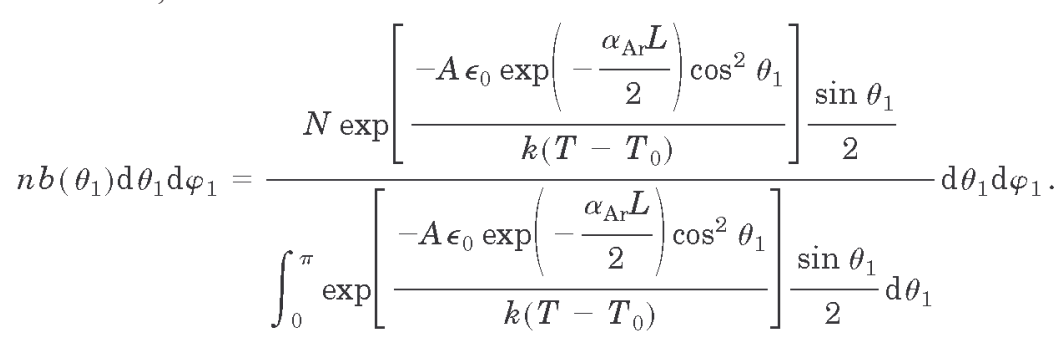

Angles $\theta$ and $\varphi$ used in Eqs. (7) and (8) are not the same as those used in Eq. (16). We must carry out a coordinate change between the coordinates of the birefringent element and those of the laboratory:

$$
\begin{aligned}
& \theta=\arccos \left(\sin \theta_{1} \sin \varphi_{1}\right), \\
& \varphi=a \cos \left[\left(\frac{1}{1+\cot ^{2} \theta_{1} \sec ^{2} \varphi_{1}}\right)^{1 / 2}\right] .
\end{aligned}
$$

Equation (8) describes the modification of the electric field of the He-Ne beam when it goes through the sample. To compare the theoretical model with the measurements, we must introduce the action of the polarizer and the analyzer as well as calculate the transmission efficiency transmission:

$$
\begin{aligned}
& \left(\begin{array}{l}
E_{x}^{\prime} \\
E_{y}^{\prime}
\end{array}\right)=\exp \left(-\frac{N \alpha_{\mathrm{He}-\mathrm{Ne}} d}{2}\right) \frac{1}{2}\left[\begin{array}{cc}
1 & -1 \\
-1 & 1
\end{array}\right] \\
& \times\left[R_{\left(-\varphi^{N}\right)} \times W_{0}^{N} \times R_{\left(\varphi^{N}\right)}\right] \\
& \times \cdots \times\left[R_{\left(-\varphi^{1}\right)} \times W_{0}^{1} \times R_{\left(\varphi^{1}\right)}\right] \\
& \times \frac{1}{2}\left[\begin{array}{ll}
1 & 1 \\
1 & 1
\end{array}\right] \times\left(\begin{array}{l}
E_{x} \\
E_{y}
\end{array}\right), \\
& \eta_{\text {tramsmission }}=\frac{\left(\begin{array}{ll}
E_{x}^{\prime} & E_{y}^{\prime}
\end{array}\right) \times\left(\begin{array}{l}
E_{x}^{\prime *} \\
E_{y}^{\prime *}
\end{array}\right)}{\left(\begin{array}{ll}
E_{x} & E_{y}
\end{array}\right) \times\left(\begin{array}{l}
E_{x}^{*} \\
E_{y}^{*}
\end{array}\right)} \text {. }
\end{aligned}
$$

With this approach it is not possible to find an analytical solution for the transmission efficiency. However, by introducing the angular distribution of the birefringent element in Eq. (18), we can carry out a numerical simulation by the Monte Carlo technique. To do this, we calculate the distribution of 
the molecule as a function of the $\theta_{1}$ angle; the distribution is discretized for each degree. Thus we have a list of numbers, each representing an angle and their sum is the number of possible states and the number of times that each angle is represented corresponds to its probability. Then we do a random drawing into this list to obtain the angle $\theta_{1}$ for the layer $k$. Variable changes as well as Jones matrices are calculated to determine the polarization state at the output of this layer

Table 1 Parameters Obtained from the Photoinduced Birefringence Fitting Polymer $\quad A / k\left[\mathrm{~K}\left(\mathrm{~mW} / \mathrm{cm}^{2}\right)^{-1 / 2}\right] T_{0}(\mathrm{~K})$

$\begin{array}{lll}\text { PVK:DMNPAA } & 27 & 229 \\ \text { C6-C11-DMNPAA } & 75 & 260 \\ \text { C11-C6-DMNPAA } & 1 & 264\end{array}$

Further runs are processed until the entire film is crossed. An average is done over ten simulations. The temperature is then changed, and calculation goes on. The latter is presented in Fig. 4. We have adjusted the parameters $A$ and $T_{0}$ to fit the different behaviors that vary with temperature. For the three polymers studied, the values of these parameters are given in Table 1.

Ordinary and extraordinary indices have been chosen to be the reasonable values of $n_{o}=1.6$ and $n_{e}=$ 1.61. The other parameters have been fixed according to the measurements done on the samples. One can see in Fig. 4 that the theoretical interpolations fit quite well the measurement for the polymers PVK: DMNPAA and C6-C11-DMNPAA, whereas the curve shapes mismatch the experimental behavior for C11-C6-DMNPAA. The reasons for this discrepancy will be detailed in Section 4.

\section{DIFFRACTION EFFICIENCY}

\section{A. Experiments}

By using the possibility of inducing birefringence in the DMNPAA doped polymer, we have written phase holograms and recorded diffraction efficiency versus temperature. The setup used to measure the diffraction efficiency is shown in Fig. 7. A more detailed view is presented in Ref. [22]. A linearly polarized argon laserbeam, with 514-nm wavelength and 3-mW/cm intensity, is extended and separated by a $50 / 50$ beam splitter. Both of the resulting beams interfere inside the sample with $10^{\circ}$ external angle and write a nonpermanent phase grating by turning the birefringent molecules. A 633-nm $\mathrm{He}-\mathrm{Ne}$ beam, with the same polarization as that of the argon beams, is directed to the sample at the Bragg angle. There it is diffracted by the phase grating to a detector. The $650-\mu \mathrm{W} / \mathrm{cm}$ intensity of the $\mathrm{He}-\mathrm{Ne}$ beam has been set so as not to disturb the molecular orientation imposed by the argon beam. The polymer film is placed on a thermal regulation system in a vacuum chamber. It is perpendicularly positioned with respect to the bisector of the argon beams.

The He-Ne beam is used as a probe; we have thus recorded, in situ, the growth of the diffraction efficiency after the opening of the argon beam shutter. To interpolate these measurements, we have used the same equation as that for the photoinduced birefringence [Eq. (1)]. However, since no light could be diffracted without a writing beam, the phase parameter $\varphi$ is always null in this case. Thus the equation that gives the maximum efficiency is simplified and becomes

$$
\eta_{\max }=\sin ^{2}(A+B) \text {. }
$$

The sensitivity of the diffraction efficiency is defined exactly as it was for the photoinduced birefringence and is given in Eq. (3).

Experimental results of diffraction efficiency versus temperature are plotted in Fig. 8 for the saturation amplitude and in Fig. 9 for the sensibility. We can see that, as for the birefringence, the temperature has a large influence over the diffraction efficiency, since the sensitivity seems not to be significantly modified.

\section{B. Mathematical Model}

The intensity of the argon beams decreases over the thickness of the sample. Therefore the index modulation achieved by the photoinduced orientation of the chro-mophores varies through the polymer. To calculate the diffraction efficiency, we have used the coupled wave theory, which allows a matrix processing in which each matrix represents a sample layer. ${ }^{[35,36]}$ According to this formulation, at the output of the sample the transmitted He-Ne wave $\left(R_{\text {out }}\right)$ and the diffracted wave $\left(S_{\text {out }}\right)$ are given by 


$$
\left(\begin{array}{l}
R_{\text {out }} \\
S_{\text {out }}
\end{array}\right)=\prod_{k=1}^{N} M_{k}\left(\begin{array}{l}
1 \\
0
\end{array}\right)
$$

$M_{k}$, the matrix describing the $k$ th-layer behavior, is defined as

$$
M_{k}=\left[\begin{array}{cc}
\left(\cos \phi+i \frac{\xi \sin \phi}{\phi}\right) \exp (-\zeta) & -i\left(\frac{C_{R}}{C_{S}}\right)^{1 / 2} \frac{\nu \sin \phi}{\phi} \exp (-\zeta) \\
-i\left(\frac{C_{R}}{C_{S}}\right)^{1 / 2} \frac{\nu \sin \phi}{\phi} \exp (-\zeta) & \left(\cos \phi-i \frac{\xi \sin \phi}{\phi}\right) \exp (-\zeta)
\end{array}\right],
$$

with

$$
\begin{aligned}
\zeta & =\frac{d}{2}\left(\frac{\alpha}{C_{R}}+\frac{\alpha}{C_{S}}+i \psi\right), \\
\xi & =i \frac{d}{2}\left(\frac{\alpha}{C_{R}}-\frac{\alpha}{C_{S}}-i \psi\right), \\
\phi & =\left(\zeta^{2}+\nu^{2}\right)^{1 / 2}, \quad \nu=\kappa d /\left(C_{R} C_{S}\right)^{1 / 2}, \\
C_{R} & =\cos \theta_{t}, \quad C_{S}=\cos \theta_{d}, \\
\kappa & =\beta \frac{\epsilon_{r 1}^{\prime}-i \epsilon_{r 1}^{\prime \prime}}{4 \epsilon_{r 0}^{\prime}}, \quad \alpha=\beta \frac{\epsilon_{r 0}^{\prime \prime}}{2 \epsilon_{r 0}^{\prime}}, \quad \beta=\frac{2 \pi}{\lambda}\left(\epsilon_{r 0}^{\prime}\right)^{1 / 2},
\end{aligned}
$$

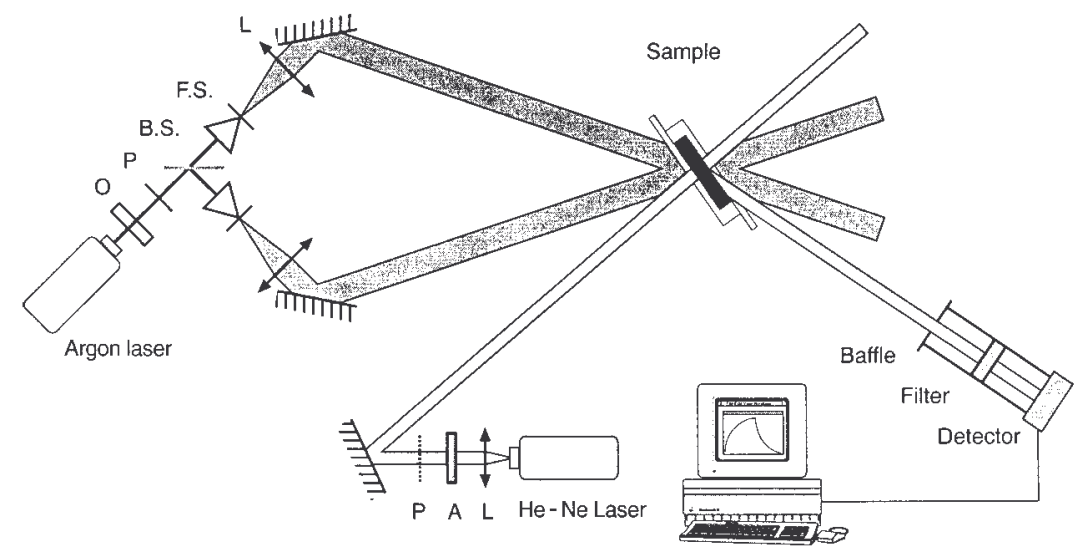

Fig. 7. Holographic setup. O, shutter; P, polarizer; B.S., beam splitter; F.S., spatial filtering; L, lens; A, attenuator.

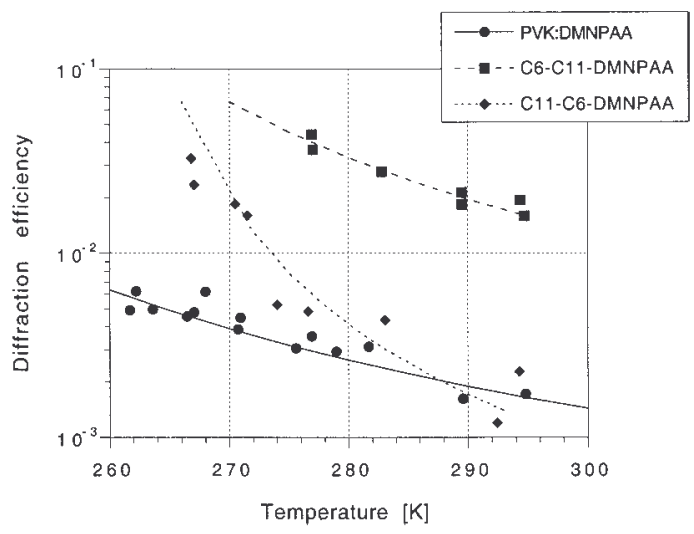

Fig. 8. Saturation amplitude of the diffraction efficiency versus temperature of the sample for the three polymers studied. Curves are interpolations by the theoretical model. 


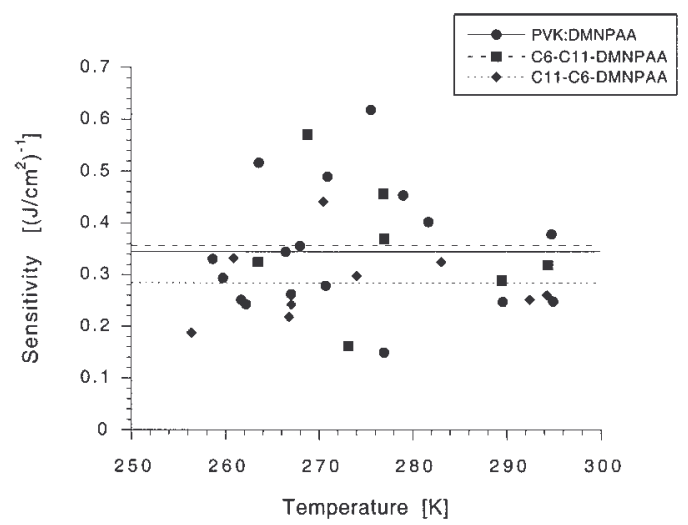

Fig. 9. Sensitivity of the diffraction efficiency versus sample temperature for the three polymers studied.

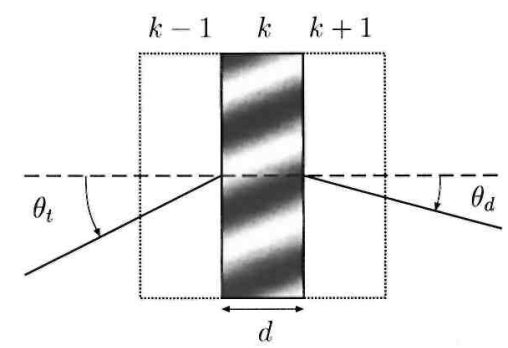

Fig. 10. Geometry and description of the parameters used in the $M_{k}$ matrix.

where $d$ is the thickness of a layer, $\varepsilon_{\mathrm{r} 0}^{\prime}$ and $\varepsilon^{\prime \prime}{ }_{r 0}$ are, respectively, the real and imaginary parts of the mean dielectric constant of the grating written in the material, $\varepsilon_{r l}^{\prime}$ and $\varepsilon_{r l}^{\prime \prime}$ are the real and imaginary parts of the dielectric constant modulation amplitude, $\theta_{t}$ and $\theta_{d}$ are the angles of the incident and diffracted beams (Fig. 10), and $\psi$ is an out-of-Bragg parameter described from the Ewald circle. In the case involved, the above equations can be greatly simplified, since we are in the Bragg condition and the setup is symmetric: $\psi=0$ and $C_{R}=C_{S}=\cos \theta_{B}\left(\theta_{t}=\theta_{d}=\theta_{B}\right)$. Moreover, we have determined by measurement that the absorption grating is negligible facing the index grating: $\varepsilon^{\prime \prime}{ }_{\mathrm{r} 1}<<\varepsilon_{r l}^{\prime}$. Equations (23) can thus be rewritten as

$$
\begin{aligned}
& \zeta=\frac{d \alpha}{\cos \theta_{B}}, \quad \xi=0, \quad \phi=\nu=\frac{\kappa d}{\cos \theta_{B}}, \\
& \kappa=\beta \frac{\epsilon_{r 1}^{\prime}}{4 \epsilon_{r 0}^{\prime}}, \quad \alpha=\beta \frac{\epsilon_{r 0}^{\prime \prime}}{2 \epsilon_{r 0}^{\prime}}, \quad \beta=\frac{2 \pi}{\lambda}\left(\epsilon_{r 0}^{\prime}\right)^{1 / 2} .
\end{aligned}
$$

Other approximations can also be made by considering the gratings ${ }^{[37]}$

$$
2 \pi n / \lambda \gg \epsilon_{r 0}^{\prime \prime}, \quad 2 \pi n / \lambda \gg \epsilon_{r 1}^{\prime \prime}, \quad \epsilon_{r 0}^{\prime} \gg \epsilon_{r 1}^{\prime},
$$

which allow us to write

$$
\begin{gathered}
\frac{1}{2} \frac{\epsilon_{r 1}^{\prime}}{\left(\epsilon_{r 0}^{\prime}\right)^{1 / 2}}=n_{1}, \\
\frac{2 \pi}{\lambda} \frac{\epsilon_{r 0}^{\prime \prime}}{\left(\epsilon_{r 0}^{\prime}\right)^{1 / 2}}=\alpha_{\mathrm{He}-\mathrm{Ne}},
\end{gathered}
$$

where $n_{l}$ is the amplitude of the index modulation and $\alpha_{\mathrm{He}-\mathrm{Ne}}$ is the absorption coefficient at the reading wavelength $(633 \mathrm{~nm})$.

By using Eqs. (24) and (26), we can redefine the matrix as follows: 


$$
\begin{aligned}
M_{k}= & \exp \left(-\frac{\alpha_{\mathrm{He}-\mathrm{Ne}} d}{2 \cos \theta_{B}}\right) \\
& \times\left[\begin{array}{cc}
\cos \left(\frac{\pi d}{\lambda \cos \theta_{B}} n_{1}\right) & -i \sin \left(\frac{\pi d}{\lambda \cos \theta_{B}} n_{1}\right) \\
-i \sin \left(\frac{\pi d}{\lambda \cos \theta_{B}} n_{1}\right) & \cos \left(\frac{\pi d}{\lambda \cos \theta_{B}} n_{1}\right)
\end{array}\right]
\end{aligned}
$$

In the text below, the following notation is used: Sample locations where the illumination pattern is at its minimum are referred to as dark zones, and sample locations where the illumination pattern is at its maximum are called lighted zones. We must now define the refractive-index modulation created by the interference of both argon beams. The illumination pattern is a cosine whose minimum is zero and whose maximum has four times the intensity of a writing beam, since both argon beams have the same intensity and polarization. Each sample layer can be decomposed into a large number of birefringent elements, themselves made up of several parallel chromophore molecules. The angular distribution of the birefringent elements can be described in the same way as that for the transmission efficiency [Eq. (16)]. In lighted zones the argon intensity depends on the sample depth. In the dark zones, since there is no light, elements are randomly oriented:

$$
\begin{aligned}
& \frac{n b_{l}\left(\theta_{1}, \varphi_{1}, L\right)}{N} \\
& \quad=\frac{\exp \left[\frac{-\mathcal{A} \epsilon(L) \cos ^{2} \theta_{1}}{k\left(T-T_{0}\right)}\right] \frac{\sin \theta_{1}}{2}}{\iint_{0}^{\pi} \exp \left[\frac{-\mathcal{A} \epsilon(L) \cos ^{2} \theta_{1}}{k\left(T-T_{0}\right)}\right] \frac{\sin \theta_{1}}{2} \mathrm{~d} \theta_{1} \mathrm{~d} \varphi_{1}},
\end{aligned}
$$

with

$$
\epsilon(L)=\left\{\begin{array}{ll}
\epsilon_{0} \exp \left(-\frac{\alpha_{\mathrm{Ar}} L}{2 \cos \theta_{B \mathrm{Ar}}}\right) & \text { for } l \text { indicating lighted zones }\left(n b_{l}\right), \\
0 \quad \forall L & \text { for } l \text { indicating dark zones }\left(n b_{l}\right)
\end{array},\right.
$$

where $\boldsymbol{\theta}_{\boldsymbol{B} A r}$ is the Bragg angle of the argon beams. The other angles and variables have the same meanings as those for the transmission efficiency calculation.

The index ellipsoid of a birefringent element, in molecular coordinates, is described by

$$
\frac{x^{2}}{n_{o}^{2}}+\frac{y^{2}}{n_{o}^{2}}+\frac{z^{2}}{n_{e}^{2}}=1 .
$$

In laboratory coordinates this molecule is oriented according to $\theta_{1}$ and $\varphi_{1}$ angles [see Fig. 6(b)]. By using the following variable change, one finds the formulation of the index ellipsoid with respect to these axes:

$$
\begin{aligned}
& x=\left(\cos \varphi_{1}\right) x_{1}-\left(\sin \varphi_{1}\right) y_{1}, \\
& y=\left(\cos \theta_{1} \sin \varphi_{1}\right) x_{1}+\left(\cos \theta_{1} \cos \varphi_{1}\right) y_{1}-\left(\sin \theta_{1}\right) z_{1} \\
& z=\left(\sin \theta_{1} \sin \varphi_{1}\right) x_{1}+\left(\sin \theta_{1} \cos \varphi_{1}\right) y_{1}+\left(\cos \theta_{1}\right) z_{1} .
\end{aligned}
$$

Indices with respect to the $y_{1}$ and $z_{1}$ axes are expressed as

$$
\begin{aligned}
& \frac{1}{n_{y_{1}}^{2}}=\frac{\sin ^{2} \varphi_{1}}{n_{o}^{2}}+\frac{\cos ^{2} \theta_{1} \cos ^{2} \varphi_{1}}{n_{o}^{2}}+\frac{\sin ^{2} \theta_{1} \cos ^{2} \varphi_{1}}{n_{e}^{2}}, \\
& \frac{1}{n_{z_{1}}^{2}}=\frac{\sin ^{2} \theta_{1}}{n_{o}^{2}}+\frac{\cos ^{2} \theta_{1}}{n_{e}^{2}},
\end{aligned}
$$

respectively.

The polarization axis of the argon beam is directed along the $z_{1}$ axis [Fig. 6(b)]. So birefringent molecules tend to have their transition axis oriented in the $x_{1}-y_{1}$ plane. Hence we can postulate that, in 
average through the film thickness, the principal axes of birefringence are oriented with respect to $y_{1}$ for the extraordinary index and with respect to $z_{1}$ for the ordinary index. Therefore the refractive indices effectively met by the reading beam are obtained by carrying out a statistical average over the molecular orientation. These are obtained by using the angular distributions written in Eq. (28), depending on whether the He-Ne beam crosses a lighted or a dark zone. Equations (33) below give the average indeces with respect to the $z_{1}$ and $y_{1}$ axes in dark and in lighted zones. For the sake of simplicity, we have suppressed the subscript 1 indexing axes and angles, so that from here on, all the equations are written in laboratory coordinates:

$$
\begin{aligned}
& n_{z_{\text {dark }}}=\iint_{0}^{\pi} \frac{\sin \theta}{2 \pi}\left[\frac{1}{\left(\sin ^{2} \theta\right) / n_{o}^{2}+\left(\cos ^{2} \theta\right) / n_{e}^{2}}\right]^{1 / 2} \mathrm{~d} \theta \mathrm{d} \varphi, \\
& n_{y_{\text {darl }}}=\iint_{0}^{\pi} \frac{\sin \theta}{2 \pi}\left[\frac{1}{\left(\sin ^{2} \varphi+\cos ^{2} \theta \cos ^{2} \varphi\right) / n_{o}^{2}+\left(\sin ^{2} \theta \cos ^{2} \varphi\right) / n_{e}^{2}}\right]^{1 / 2} \mathrm{~d} \theta \mathrm{d} \varphi, \\
& n_{z_{\text {lighted }}}=\iint_{0}^{\pi} \frac{\exp \left[-A \epsilon(d) \cos ^{2} \theta / k\left(T-T_{0}\right)\right](\sin \theta) / 2}{\iint_{0}^{\pi} \exp \left[-A \epsilon(d) \cos ^{2} \theta / k\left(T-T_{0}\right)\right](\sin \theta) / 2 \mathrm{~d} \theta \mathrm{d} \varphi} \\
& \times\left[\frac{1}{\left(\sin ^{2} \theta\right) / n_{o}^{2}+\left(\cos ^{2} \theta\right) / n_{e}^{2}}\right]^{1 / 2} \mathrm{~d} \theta \mathrm{d} \varphi, \quad(33 \mathrm{c}) \\
& n_{y_{\text {lighted }}}=\iint_{0}^{\pi} \frac{\exp \left[-A \epsilon(d) \cos ^{2} \theta / k\left(T-T_{0}\right)\right](\sin \theta) / 2}{\iint_{0}^{\pi} \exp \left[-A \epsilon(d) \cos ^{2} \theta / k\left(T-T_{0}\right)\right](\sin \theta) / 2 \mathrm{~d} \theta \mathrm{d} \varphi} \\
& \times\left[\frac{1}{\left(\sin ^{2} \varphi+\cos ^{2} \theta \cos ^{2} \varphi\right) / n_{o}+\left(\sin ^{2} \theta \cos ^{2} \varphi\right) / n_{e}^{2}}\right]^{1 / 2} d \theta d \varphi .
\end{aligned}
$$

Figure 11 shows the variation of the different indices of refraction with the temperature parameter $T-T_{0}$. For this calculation the ordinary index has been fixed to 1.6, and the extraordinary index has been set at 1.62. In dark zones there is no light to redirect the chromophores; thus the material is isotropic, i.e., indices with respect to $y$ and $z$ axes must be identical to each other. It can be verified that in spite of their different formulations, Eqs. (33a) and (33b) give the same numerical result. One can see that when temperature is equal to $T_{0}$, the index along $z$ in lighted zones is equal to the ordinary index. Indeed, in this configuration, all the birefringent elements are oriented perpendicularly to the argon beam polarization. Thus the $z$ axis makes a $90^{\circ}$ angle with the optical axis of the elements; this prevents the He-Ne light from seeing the extraordinary index. On the other hand, for temperature increasing to infinity, indices of the lighted zones tend toward the dark-zone index. This is because thermal agitation randomizes the molecular orientation; for high temperature the sample becomes isotropic, and it cannot produce light diffraction any longer.

Since the propagation media are birefringent, the linearly polarized $\mathrm{He}-\mathrm{Ne}$ beam can be divided into two components: ordinary and extraordinary. 


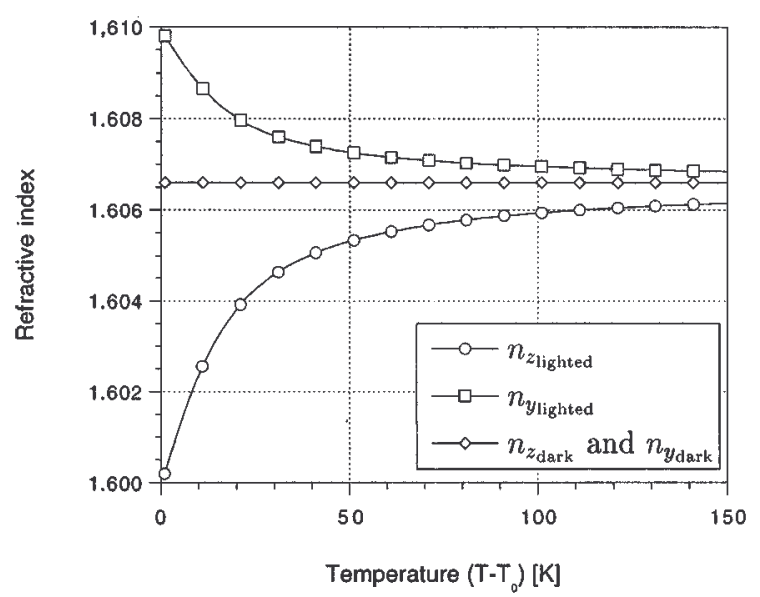

Fig. 11. Variation of the indices of refraction with the difference between temperature and temperature threshold. Circles: refractive index along the z laboratory axis in sample lighted zones, squares: refractive index along the y laboratory axis in sample lighted zones, diamonds: refractive index along the $z$ and $y$ laboratory axes in sample dark zones. The plot is based on Eq. (33).

The intensity fraction content in one or the other beam depends on $\rho$, the angle between the initial polarization of the He-Ne beam and the $z$ axis. The intensities with respect to the different axes are expressed as

$$
\begin{aligned}
& I_{z}=I_{\text {incident }} \cos ^{2} \rho, \\
& I_{y}=I_{\text {incident }} \sin ^{2} \rho .
\end{aligned}
$$

The diffraction efficiency can also be expressed in two parts: The first is related to the beam fraction subject to the $n_{z}$ indices, and the second is related to the fraction subject to the $n_{y}$ indices. The refractive-index modulation created by the interference of the argon beams and used in Eq. (27) is thus composed of the following elements:

$$
\begin{aligned}
& n_{1 z}=\frac{n_{z_{\text {lighted }}}-n_{z_{\text {dark }}}}{2}, \\
& n_{1 y}=\frac{n_{y_{\text {lighted }}}-n_{y_{\text {dark }}}}{2} .
\end{aligned}
$$

Figure 8 shows the numerical calculation of diffraction efficiency versus temperature for an initially vertically polarized He-Ne beam. This is not a Monte Carlo simulation, since the equations of the refractive indices allow a direct numerical calculation and already take into account the angular distribution of the chromophores. The indices thus obtained are representative of the experiment because measurements are done on large surfaces (comparing the molecular dimension) that average the local distributions.

For the polymers PVK:DMNPAA and C6-C11-DMNPAA, the value of the parameters $A / k$ and $T_{0}$, introduced for the photoinduced birefringence, has been reused with success to fit the experimental data of diffraction efficiency. However, for C11-C6-DMNPAA the value of $A / k$ has been changed from $1 \mathrm{~K}(\mathrm{~mW} / \mathrm{cm})$ to $10 \mathrm{~K}(\mathrm{~mW} / \mathrm{cm})^{-1 / 2}$ in order to interpolate the diffraction measurements. We also recall that for this polymer there was a discrepancy between the photoinduced birefringence data and the mathematical calculation.

\section{DISCUSSION AND CONCLUSIONS}

By studying the thermal behavior of the photoinduced birefringence, we have found that the resulting transmission efficiency drastically increases when the temperature decreases. By assuming that this property is due to the thermal agitation that randomizes the chromophore alignment, we have proposed a theoretical model. The numerical calculation arising out of this model matches the experimental results. The number of free parameters for data fitting was only 2; the other theoretical variables have been fixed to reasonable values in relation to the physical problem.

One could expect to find different behaviors depending on whether the polymer is frozen or not. However, the $T_{g}$ of the compound does not seem to have an important role in molecular orientation, since no discontinuity has been found in experimental curves around its value. Nevertheless, a temperature threshold $T_{0}$ has been intro-duced into the theoretical model. The meaning of this threshold 
is that, under this temperature value, the ther-mal agitation could no longer disturb the molecular orientation imposed by the writing beam. This implies that, from this threshold, all birefringent molecules are per-fectly oriented and, furthermore, decreasing the tempera-ture does not influence the angular distribution. This can be explained by the free-volume theory, which fore-sees that the volume between polymer strings is decreas-ing with the temperature. ${ }^{[38]}$ The chromophore molecules that occupy this volume need space to rotate. When free volume reduces below the space needed by molecules for thermal misalignment, the thermal agitation no longer influences the angular distribution. ${ }^{[30]}$ It would be inter-esting to observe the experimental behavior of the sample at this temperature threshold; but, in spite of many im-provements, the thermal stability of the sample under $250 \mathrm{~K}$ could not be ensured in our setup. Indeed, as the sample is placed in a vacuum chamber, it cannot dissipate the thermal energy provided by the argon beam that warms the sample during the writing process.

As already reported by other authors, ${ }^{[10,16,18]}$ the time constants of the transmission efficiency increase as the temperature decreases (see Fig. 12). Nevertheless, in Fig. 5 it appears that sensitivity does not vary significantly over the temperature range studied. So the change in the time constants seems to be more a calculation artifact than a reflection of the real behavior of the polymers. By using the Williams-Landel-Ferry theory ${ }^{[33]}$ and the results of Eisenbach, ${ }^{[34]}$ we have developed further the dynamics of the photoinduced birefringence in another paper. ${ }^{[39]}$

In azo dye doped polymers, transmission efficiency and diffraction efficiency result from the same phenomenon: photoinduced orientation; so it is not surprising to find the same thermal behavior for both kinds of experiments. Except for C11-C6-DMNPAA, the calculation presented in Fig. 8 has been done with the same parameters as those used to interpolate the transmission efficiency (Fig. 4). Since both calculations fit quite well the experimental data, we can conclude that there is compatibility between the models and that the parameters can be used to describe polymer sample properties. This gives us a method to objectively compare the compounds.

With the use of this method, experiments are planned to compare the threshold temperature value and the $T_{g}$ of the different compounds doped with different amounts of plasticizer.

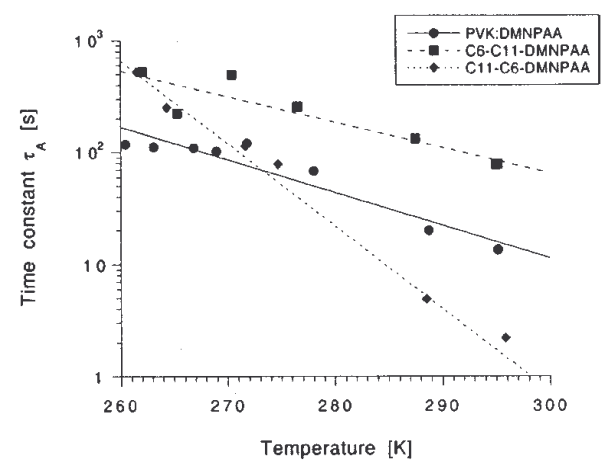

(a)

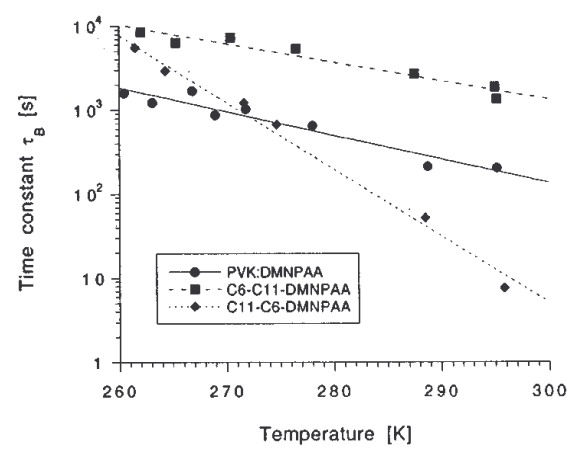

(b)

Fig. 12. Thermal dependence of both time constants of the transmission efficiency: (a) fast time constant and (b) slow time constant. The lines are data interpolations; they are guides for the eye and do not come from any model.

As was done here, the parameter will be compared with the molecular index anisotropy by doping the matrices with various azo dye chromophores.

The case of the C11-C6-DMNPAA polymer is quite worrisome: The behavior of the photoinduced birefringence cannot be reproduced by the theoretical model, and the diffraction efficiency is far higher 
than that predicted. Indeed, the parameter $A / k$ is underevaluated by a factor of 10 from the photoinduced birefringence interpolation. Since the diffraction efficiency values cannot be explained by the reorientation of the chromophores, we have searched for another mechanism responsible for this enhancement. He-Ne diffracted intensity as a function of the polarization of the writing beams as well as dichroism measurements have revealed that the grating in the C11-C6-DMNPAA polymer is mainly formed by the isomeriza-tion of the molecules from trans-to-cis configuration. The rotation of the chromophores is negligible ${ }^{[40,41]}$ Thus our models cannot be applied to this polymer. The increase of the efficiencies with time is probably related, in this case, to the lifetime of the cis state, which can vary with temperature. ${ }^{[18]}$

We have done some of our experiments with PVK polymer film containing $15 \mathrm{wt} . \%$ of DMNPAA and 30 wt. $\%$ of $N$-ethylcarbazole. This compound, with a small amount (1 wt.\%) of trinitrofluorenone, is well-known to be a good photorefractive polymer. ${ }^{[19,20]}$ In photorefractive media DMNPAA is used as nonlinear optical molecules that locally change the index of refraction of the sample. This happens through the Pockels effect in the electric space-charge field created by charge-carrier migration in the polymer. ${ }^{[42]}$ In this way photorefractivity and photo-orientation seem to be two completely different mechanisms. However, it has been discovered that photore-fractive polymers with low $T_{g}$ (below laboratory temperature) can enhance their diffraction efficiency by aligning nonlinear optical molecules in the space-charge field. ${ }^{[43]}$ Moreover, recent experiments tend to prove that the index change in PVK:DMNPAA is principally due to the birefringence of the DMNPAA molecules instead of their nonlinear response. ${ }^{[1,44]}$ In these conditions photore-fractivity and photo-orientation are quite similar, since both arise by alignment of the birefringent chromophores in the electric field: space charge and laser light, respec-tively. So it is possible that our results, developed here for photoinduced orientation, can be adapted to photore-fractivity experiments.

However, it should be noted that, in photorefractivity, the generation of the space-charge field will certainly also be affected by the temperature, since charge photogenera-tion and displacement could depend on temperature. The sensitivity could also be affected with regard to charge mobility. Thus the behavior should certainly be more complicated for photorefractivity than for photo-orientation. Further experiments are going to be done to confirm such a hypothesis.

\section{REFERENCES}

[1]. P. M. Lundquist, R. Wortmann, C. Geletneky, R. J. Twieg, M. Jurich, V. Y. Lee, C. R. Moylan, and D. M. Burland, "Organic glasses: a new class of photorefractive materials," Science 274, 1182-1184 (1996).

[2]. J. Xu, G. Zhang, Q. Wu, Y. Liang, S. Liu, X. Chen, and Y. Shen, "Holographic recording and light amplification in doped polymer film,'’ Opt. Lett. 20, 504-506 (1995).

[3]. S. V. O'Leary, "Real-time processing by degenerate four-wave mixing in polarization sensitive dye-impregnated polymer films,', Opt. Commun. 104, 245-250 (1994).

[4]. L. R. Dalton, A. W. Harper, B. Wu, R. Ghosn, J. Laquin-danum, Z. Liang, A. Hubble, and C. Xu, “Polymeric electro-optic modulators: materials synthesis and processing," Adv. Mater. 7, 519-540 (1995).

[5]. B. L. Volodin, B. Kippelen, K. Meerholz, B. Javidi, and N. Peyghambarian, “'A polymeric organical pattern recognition system for security verification,'” Nature (London) 383, 58-60 (1996).

[6]. P. M. Lundquist, C. Poga, R. G. De Voe, Y. Jia, W. E. Mo-erner, M.-P. Bernal, H. Coufal, and R. K. Grygier,

"Holographic digital data storage in a photorefractive polymer,', Opt. Lett. 21, 890-892 (1996).

[7]. M. S. Ho, A. Natansohn, and P. Rochon, “Azo polymers for reversible optical storage. 7. The effect of the size of the photochromic groups," Macromolecules 28, 6124-6127 (1995).

[8]. T. Todorov, L. Nikolova, and N. Tomova, "Polarization holography. 1: A new high-efficiency organic material with reversible photoinduced birefringence,” Appl. Opt. 23, 4309-4312 (1984).

[9]. T. Buffeteau and M. Pézolet, "In situ study of photoinduced orientation in azopolymers by time-dependent polarization modulation infrared spectroscopy," Appl. Opt. 50, 948-955 (1996).

[10]. A. M. Makushenko, B. S. Neporent, and O. V. Stolbova, "Reversible orientation photodichroism and photoisomer-ization of aromatic azo compounds. I: Model of the system,' Opt. Spectrosc. (USSR) 31, 295-299 (1971).

[11]. L. Nikolova, P. Markovsky, N. Tomova, V. Dragostinova, and N. Mateva, "'Optically-controlled photo-induced birefringence in photo-anisotropic materials,'” J. Mod. Opt. 35, 1789-1799 (1988).

[12]. P. Rochon, J. Gosselin, A. Natansohn, and S. Xie, "Optically induced and erased birefringence and dichroism in azoaromatic polymers,'’ Appl. Phys. Lett. 60, 4-5 (1992).

[13]. P. A. Blanche, Ph. C. Lemaire, C. Maertens, P. Dubois, and R. Je'ro`me, "'Polarised light induced birefringence in azo dye doped polymer: a new model and polarised holographic experiments,'” Opt. Commun. 139, 92-98 (1997).

[14]. M. Dumont, "A common model for optical ordering of pho-toisomerizable molecules,'” in Photorefractive Organic Materials. Science and Applications, F. Kajzar, V. M. Agranov-ich, and C. Y.-C. Lee, eds. (Kluwer Academic, Amsterdam, 1996), Vol. 9, pp. 501-511.

[15]. L. Nikolova, T. Todorov, N. Tomova, and V. Dragostinova, "Polarization-preserving wavefront reversal by four-wave mixing in photoanisotropic materials,'” Appl. Opt. 27, 1598- 1602 (1988).

[16]. J. S. Hwang, G. J. Lee, and T. K. Lim, “Temperature dependence of photo-induced anisotropy of azo-doped polymer film at the glass transition region of a polymer matrix,' J. Korean Phys. Soc. 27, 392-395 (1994).

[17]. S. Ivanov, I. Yakovlev, S. Kostromin, and V. Shibaev, "Laser-induced birefringence in homeotropic films of photochromic comb-shaped liquid-crystalline copolymers with azobenzene moieties at different temperatures,' Makromol. Chem. 12, 709-715 (1991).

[18]. O.-K. Song, C. H. Wang, and M. A. Pauley, “'Dynamic processes of optically induced birefringence of azo compounds in 
amorphous polymers below $T_{g}$, , Macromolecules 30, 6913-6919 (1997).

[19]. B. Kippelen, N. Peyghambarian, S. R. Lyon, A. B. Padias, and H. K. Hall, Jr., "New highly efficient photorefractive polymer composite for optical storage and image-processing applications,'” Electron. Lett. 29, 1873-1874 (1993).

[20]. K. Meerholz, B. L. Volodin, Sandalphon, B. Kippelen, and N. Peyghambarian, “A photorefractive polymer with high optical gain and diffraction efficiency near 100\%," Nature (London) 371, 497-500 (1994).

[21]. C. Maertens, P. Dubois, R. Jérôme, P.-A. Blanche, and P. C. Lemaire, "Synthesis and polarized light induced birefringence of new polymethacrylates containing carbazolyl and azobenzene pendant groups,' J. Polym. Sci., Part B: Polym. Phys. 38, 205-213 (2000).

[22]. P. A. Blanche, Ph. C. Lemaire, C. Maertens, P. Dubois, and R. Jérôme, "Temperature variation of the photoinduced birefringence of an azo dye doped polymer,' Polym. Eng. Sci. 38, 406-412 (1999).

[23]. L. Lamarre and C. S. P. Sung, "Studies of physical aging and molecular motion by azochromophorric labels attached to the main chains of amorphous polymers," Macromol-ecules 16, 1729-1736 (1983).

[24]. Y. Atassi, J. A. Delaire, and K. Nakatani, “'Coupling between photochromism and second-harmonic generation in spiropyran- and spirooxazin-doped polymer films," J. Appl. Chem. 99, 16320-16326 (1995).

[25]. M. Dumont, G. Froc, and S. Hosotte, “Alignment and orientation of chromophores by optical pumping," Nonlinear Opt. 9, 327-338 (1995).

[26]. A. Yariv and P. Yeh, Optical Waves in Crystals (Wiley, New York, 1984), pp. 121-154.

[27]. Z. Sekkat, J. Wood, and W. Knoll, "Reorientation mechanism of azobenzenes within the Tran $\rightarrow$ Cis photoisomerization," J. Phys. Chem. 99, 17226-17234 (1995).

[28]. T. G. Pedersen, P. M. Johansen, N. C. R. Holme, P. Ra-manujam, and S. Hvilsted, “Theoretical model of photoin-duced anisotropy in liquid-crystalline azobenzene side-chain polyesters,'” J. Opt. Soc. Am. B 15, 1120-1129 (1998).

[29]. I. Mita, K. Horie, and K. Hirao, "Photochemistry in polymer solids. 9. Photoisomerization of azobenzene in a polycarbonate film,'” Macromolecules 22, 558-563 (1989).

[30]. S. Xie, A. Natansohn, and P. Rochon, "Recent development in aromatic azo polymers research,', Chem. Mater. 5, 403411 (1993)

[31]. Z. Sekkat and M. Dumont, "Photoinduced orientation of azo dyes in polymeric films. Characterization of molecular angular mobility,' Synth. Met. 54, 373-381 (1993).

[32]. N. G. McCrum, B. E. Read, and G. Williams, Anelastic and Dielectric Effects in Polymeric Solids (Wiley, New York, 1967), pp. 169-174.

[33]. M. L. Williams, R. F. Landel, and J. D. Ferry, “The temperature dependence of relaxation mechanisms in amorphous polymers and other glass-forming liquids,'” J. Am. Chem. Soc. 77, 3701-3707 (1955).

[34]. C. D. Eisenbach, "Effect of polymer matrix on the cis-trans isomerization of azobenzene residues in bulk polymers," Makromol. Chem. 179, 2489-2506 (1978).

[35]. D. Kermisch, "Nonuniform sinusoidally modulated dielectric gratings,', J. Opt. Soc. Am. 59, 1409-1414 (1969).

[36. L. B. Au, J. C. W. Newell, and L. Solymar, "Non-uniformities in thick dichromated gelatin transmission gratings," J. Mod. Opt. 34, 1211-1225 (1987).

[37]. H. Kogelnik, "Coupled wave theory for thick hologram grating,' Bell Syst. Tech. J. 48, 2909-2947 (1969).

[38]. R. N. Haward, The Physics of Glassy Polymers (Wiley, New York, 1973), pp. 25-41 and 171-176.

[39]. C. Maertens, P. Dubois, R. Je'ro^me, P.-A. Blanche, and Ph. C. Lemaire, "Dynamics of the photoinduced orientation and relaxation of new polymethacrylates containing carbazolyl and azobenzene pendant groups,' Polym. Int. 48, 205-211 (1999).

[40]]. P. A. Blanche, Ph. C. Lemaire, C. Maertens, P. Dubois, and R. Je'ro^me, "Polarization holography reveals the nature of the grating in azo-dye contained polymers," J. Opt. Soc. Am. B (to be published).

[41]. P.-A. Blanche, P. C. Lemaire, M. Dumont, and M. Fischer, "Photoinduced orientation of azo dye in various polymer matrices,'” Opt. Lett. 24, 1349-1351 (1999).

[42]. S. Ducharme, J. C. Scott, R. J. Twieg, and W. E. Moerner, "Observation of the photorefractive effect in a polymer,' Phys. Rev. Lett. 66, 1846-1849 (1991).

[43]. W. E. Moerner, S. M. Silence, F. Hache, and G. C. Bjork-lund, "'Orientationally enhanced photorefractive effect in polymer,' J. Opt. Soc. Am. B 11, 320-330 (1994).

[44]. W. E. Moerner, A. Grunnet-Jepsen, C. L. Thompson, and R. J. Twieg, "Mechanisms of photorefractivity in polymer composites," in Organic Photorefractive Materials and Xerographic Photoreceptors, S. Ducharme and J. W. Stasiak, eds., Proc. SPIE 2850, 2-13 (1996). 